\title{
Tratamiento quirúrgico de la hemorragia intracerebral espontánea. Parte II: Hemorragia infratentorial
}

\author{
A. Pérez-Núñez; R. Alday; J.J. Rivas; A. Lagares; P.A. Gómez; J.F. Alén; I. Arrese y R.D. Lobato.
}

Servicio de Neurocirugía. Hospital Universitario Doce de Octubre. Madrid.

Resumen

El tratamiento quirúrgico juega un papel fundamental en el manejo de algunos pacientes con hematomas de cerebelo, y sin embargo, no existe una guía de tratamiento universalmente aceptada que permita seleccionar a este subgrupo de pacientes.

El objetivo del presente trabajo fue revisar la base sobre la que se fundamentan las indicaciones del tratamiento quirúrgico en esta patología.

En ausencia de ensayos clínicos que afronten este problema, las series clínicas muestran que los criterios más consistentes para la decisión terapéutica son el nivel de consciencia, el tamaño del hematoma, la presencia de hidrocefalia y los datos radiológicos de compresión de los espacios continentes de LCR en la fosa posterior. El parámetro mejor estudiado como reflejo de este último aspecto posiblemente sea la deformidad del IV ventrículo. La literatura sugiere que los hematomas de 4 o más $\mathrm{cm}$ de diámetro, o que causan una oclusión completa del IV ventrículo o de la cisterna prepontina deben ser intervenidos independientemente del nivel de consciencia, al presentar una compresión significativa del tronco del encéfalo (TDE). Por el contrario, es probable que hematomas de menos de $3 \mathrm{~cm}$ y que no deforman el IV ventrículo, no causen una compresión importante en la fosa posterior, y puedan ser manejados de forma conservadora o mediante el drenaje de la hidrocefalia si fuera preciso. Para hematomas de tamaño intermedio la decisión terapéutica está menos clara, pudiendo optarse por observación estricta en los pacientes con GCS 14-15 o con drenaje ventricular externo (DVE) aislado en aquellos con GCS $<14$ que presenten hidrocefalia. En presencia de un bajo nivel de consciencia a pesar del tratamiento de la hidrocefalia, 0 en ausencia de ésta, se debería realizar una evacuación del hematoma.

Finalmente, no parece indicado el tratamiento de pacientes con GCS 3 y ausencia de reflejos de tronco, o

Recibido: 25-07-07. Aceptado 1-10-07 aquéllos en los que por su edad avanzada o mala calidad de vida previa presenten un pronóstico funcional malo. Se ha encontrado además que los pacientes en coma y con signos radiológicos de grave compresión del TDE las posibilidades de una buena recuperación son muy escasas.

A pesar de todo el tratamiento ha de ser individualizado en cada caso, ya que no existe la evidencia suficiente que permita elaborar una guía de aplicación estricta.

PALABRAS CLAVE: Hematoma de cerebelo. Tratamiento. Cirugía. Hemorragia intracerebral.

Surgical treatment for spontaneous intracerebral haemorrhage. Part II: Infratentorial haematomas

\section{Summary}

Surgery plays a mayor role in the management of some patients with cerebellar haematomas, although a universally accepted treatment guideline is lacking.

The aim of this study was to review the existing evidence supporting surgical evacuation of the haematoma in this pathology.

Without any clinical trial on this field, data derived from clinical series suggest that the level of consciousness, the size of the haematoma, the presence of hydrocephalus and the compression of the posterior fossa CSF containing spaces are the main criteria to decide management. Fourth ventricular compression seems to be the best indicator of the last parameter. Existing bibliography shows that haematomas greater than $4 \mathrm{~cm}$ or causing complete obliteration of the fourth ventricle or prepontine cistern need surgical evacuation irrespective of the level of consciousness, as they indicate a significant compression of the brainstem. On the other hand, it seems that haematomas of less than $3 \mathrm{~cm}$ and without fourth ventricular compression can be managed conservatively or by means of ventricular drainage if hydrocephalus exists and requires treatment. The 
management of intermediate sized haematomas is less clear although conservative approach could be adopted in presence of adequate neurological status, with EVD in the case of hydrocephalus with low consciousness level. If the level of consciousness is low despite the treatment of hydrocephalus, or in absence of this latter, haematoma evacuation is indicated.

Finally, patients with flaccid tetraplejia and absent oculocephalic reflexes, and those whose age or basal condition precludes an adequate functional outcome are not suitable for aggressive treatment. Moreover, some studies have shown that comatose patients with CT scan evidence of severe brainstem compression present a reduced probability of good outcome.

Anyway, management should be decided on an individual basis, as there is no enough evidence to support a strict treatment protocol.

KEY WORDS: Cerebellar haematoma. Surgery. Treatment. Intracerebral haemorrhage.

\section{Introducción}

La hemorragia intracerebral espontánea (HIE) en la fosa posterior asienta fundamentalmente en la protuberancia y el cerebelo. En general se acepta que el tratamiento de la hemorragia primaria troncoencefálica es no quirúrgico $2,6,8,11,21,22$, por lo que este trabajo se centra en los hematomas de cerebelo.

El hematoma de cerebelo representa aproximadamente un $10 \%$ de las HIE, se observa fundamentalmente entre la $6^{\mathrm{a}}$ y $8^{\mathrm{a}}$ décadas de la vida y muestra cierto predominio en el género masculino $1,4,7,9,12,13,15,19,27,28$. Parece que, al igual que en la localización supratentorial, el daño vascular producido por la HTA crónica representa el factor etiológico fundamental, estando implicado en el $70 \%$ de los $\operatorname{casos}^{3,4,7,9,12-14,23,26}$. Las siguientes causas en frecuencia las constituyen los trastornos de la coagulación, las malformaciones vasculares y los tumores ${ }^{3,4,23}$. Aunque la angiopatía amiloide se ha encontrado en un $14 \%$ de las los casos en series autópsicas, su papel en las series clínicas parece marginal, y como en la patología supratentorial, de predominio en personas ancianas ${ }^{4,12}$. Muchos de estos pacientes (15$51,3 \%$ ) presentan antecedentes de ictus previos ${ }^{4,12}$. La localización más frecuente de estos hematomas es la región de los núcleos dentados (es rara la hemorragia en vermis), que habitualmente afectan al territorio de la arteria cerebelosa superior y es infrecuente la extensión al tronco del encéfalo (TDE) $)^{9,12,23}$.

Clásicamente los hematomas cerebelosos fueron considerados un subtipo de HIE con un pronóstico especialmente gris $^{3,9,26}$. En las series recientes la mortalidad oscila entre $18-58 \%$, y entre el 50 y el $90 \%$ de los supervivientes presentan una buena recuperación funcional, con una vida independiente $3,4,5,7,12,17,27,30$. Estos datos reflejan que la mortalidad puede ser similar a la HIE supratentorial y que el pronóstico funcional podría ser incluso mejor. Los factores que han mostrado influencia en la evolución son fundamentalmente el grado clínico, el volumen del hematoma y la presencia de hidrocefalia ${ }^{3-5,7,10,12,17,20,23,26,30}$. Algunos autores han encontrado valor pronóstico para los signos clínicos de compresión del TDE, el grado de compresión de la cisterna cuadrigeminal, la deformidad del IV ventrículo, la oclusión de las cisternas perimesencefálicas, la hemorragia intraventricular, la existencia de comorbilidad significativa y existe controversia en cuanto al papel que juega la edad ${ }^{4,5,7,10,12,26,27,30}$. El hematoma cerebeloso se caracteriza en la mayoría de los casos por una clínica de instauración brusca y curso progresivo de cefalea, mareo, vómitos e inestabilidad, asociada a una disminución más o menos importante del nivel de consciencia y un cuadro cerebeloso más o menos florido 9 . La presentación depende de diversos factores que pueden presentarse con intensidad variable y aislados o combinados entre sí: el grado de compresión de la fosa posterior (y fundamentalmente del TDE), la hidrocefalia asociada, la existencia de un infarto de cerebelo subyacente al hematoma o el desarrollo secundario de un infarto de $\mathrm{TDE}^{5,10,12}$. Esta variabilidad condiciona la existencia de dos tipos básicos en los que se puede agrupar a la mayoría de los pacientes ${ }^{14,20,30}$. El primero representa a aquellos pacientes en los que lo más llamativo es la afección del nivel de consciencia, debutando bien con una pérdida brusca inicial o bien con un deterioro progresivo en la misma. En éstos, el desarrollo más o menos precoz de compresión del TDE y/o la hidrocefalia son los principales factores patógenos implicados, y los signos cerebelosos pasan desapercibidos. El segundo grupo de pacientes presentan un curso clínico más benigno, con afectación leve o ausente del nivel de consciencia, domina el cuadro un síndrome cerebeloso y los signos de afección del TDE son leves o ausentes.

Aproximadamente un tercio de los pacientes se presenta en coma, y es reconocida la posibilidad de un deterioro brusco del nivel de consciencia en pacientes conscientes, impredecible desde un punto de vista exclusivamente clínico $^{7,9,10,12,20,23}$. A diferencia del hematoma supratentorial, el crecimiento del sangrado parece infrecuente en los hematomas de cerebelo, habiendo sido reportado en sólo $2,7-9 \%$ de los $\operatorname{casos}^{4,29}$. Por ello, aunque el tamaño del hematoma puede aumentar, el deterioro clínico debe atribuirse más probablemente al desarrollo de edema, la progresión de la hidrocefalia, los fenómenos de herniación o la aparición de isquemia troncoencefálica secundaria ${ }^{10,12}$.

Se acepta tradicionalmente que el tratamiento quirúrgico juega un papel fundamental en el manejo de los hematomas de cerebelo, aunque sin embargo algunos 
estudios reflejan cierta variabilidad en el manejo y en los criterios empleados para la indicación quirúrgica en estos pacientes $^{4,5,12,13,27,29}$. El objetivo de esta revisión es la identificación de la base científica sobre la que se fundamenta la indicación de cirugía en estos casos, y si es posible, la elaboración de un protocolo de decisión terapéutica.

\section{Material y método}

Se empleó la base de datos PUBMED para la búsqueda de artículos relativos al manejo quirúrgico de los hematomas de cerebelo. Se buscaron ensayos clínicos, meta-análisis, revisiones o series de casos encaminados a determinar los criterios necesarios para la indicación del tratamiento quirúrgico. Las palabras clave empleadas fueron "cerebellar hematoma" y "cerebellar hemorrhage" combinadas con "surgery", "treatment" y "management".

\section{Resultados}

No hemos encontrado ensayos clínicos que evalúen la utilidad del tratamiento quirúrgico en los hematomas de cerebelo. Existen diversas series clínicas que analizan el papel de la evacuación del hematoma en esta patología. En los estudios previos al desarrollo de la TAC el interés radicaba fundamentalmente en el reconocimiento de este cuadro y del hecho de que la cirugía podía ofrecer una alternativa de supervivencia a pacientes que de otro modo tendrían un pronóstico infausto ${ }^{19,27}$. Más allá de la dificultad para reconocer la existencia de un hematoma de cerebelo, las indicaciones quirúrgicas podían casi exclusivamente fundamentarse en criterios clínicos. Tras la aparición de la TAC, gracias a la mejora en el diagnóstico de los hematomas de fosa posterior, se han publicado numerosos trabajos que hacen referencia a los resultados del tratamiento de estos pacientes y que analizan los factores implicados en la evolución, algunas de las cuales se pueden destacar por su interés en la delimitación de las indicaciones del tratamiento quirúrgico:

- En 1978 Little et al. ${ }^{14}$ describieron la asociación entre un diámetro del hematoma mayor de $3 \mathrm{~cm}$ y la presencia de datos clínicos de compresión del TDE. En una serie de 10 pacientes, 6 se presentaron con deterioro del nivel de consciencia, de los cuales 5 presentaban hematomas de más de $3 \mathrm{~cm}$ y todos mostraban en la TAC hidrocefalia moderada o grave. Tres de estos pacientes mostraban hemorragia intraventricular asociada. En este grupo se desestimó el tratamiento en uno de los pacientes que finalmente falleció. De los 5 pacientes intervenidos (evacuación del hematoma) cuatro recuperaron una vida independiente y uno falleció. Ninguno de los 4 pacientes con un comportamiento clínico más benigno precisó intervención quirúrgica experimentando buena evolución. Los hematomas diagnosticados en estos últimos pacientes midieron en todos los casos menos de $3 \mathrm{~cm}$ y no se observó hidrocefalia en ninguno.

- En 1986 Weisberg ${ }^{32}$ introdujo el término "tight posterior fossa", al que aquí nos referiremos como "compresión de fosa posterior". Revisando una serie de 20 pacientes definieron el mismo como la presencia en TAC de borramiento de las cisternas basales de la fosa posterior y dilatación de los ventrículos supratentoriales, con borramiento inconstante del IV ventrículo. Encontraron estos datos en 14 pacientes (el IV ventrículo aparecía ocluido en 10 y lleno de sangre en 4), con hematomas entre 3 y $4,7 \mathrm{~cm}$. Todos estos pacientes mostraron deterioro neurológico, en 6 de ellos hasta el coma. Los 6 pacientes comatosos no mejoraron tras tratamiento médico de la hipertensión intracraneal y fallecieron, en tanto que los 8 restantes fueron intervenidos y sobrevivieron, recuperando deambulación independiente 6 de ellos. De los 6 pacientes que no presentaron signos de "compresión de la fosa posterior", con hematomas entre 2,6-3,6 cm, ninguno experimentó deterioro, sobreviviendo y recuperando la deambulación todos ellos (2 con apoyo). El autor terminó por concluir que los signos de "compresión de la fosa posterior" eran el mejor criterio para la indicación terapéutica.

- Taneda et $\mathrm{al}^{29}$, basándose en la hipotética relación entre el efecto de masa de lesiones de fosa posterior y la deformidad de la cisterna cuadrigeminal en la TAC, estudiaron la relación de este último rasgo con el pronóstico clínico y su utilidad como criterio de decisión terapéutica en pacientes con hematomas de cerebelo. Revisaron retrospectivamente una serie consecutiva de 75 pacientes y clasificaron la morfología cisternal en grado I (normal), grado II (deformada) y grado III (colapsada). Registraron también la existencia de hidrocefalia y el tamaño del hematoma (que calcularon mediante el producto del máximo diámetro transversal por el máximo diámetro perpendicular a éste)) y analizaron la relación entre estos factores y el tipo de tratamiento y la evolución. Encontraron que el $97 \%$ de los pacientes con una morfología cisternal clasificada como grado I evolucionaron favorablemente si no había hidrocefalia o si ésta era tratada en menos de 48 horas. Cuando en los pacientes con grado I e hidrocefalia ésta se trató más allá de 48 horas, sólo un 47\% tuvieron una evolución adecuada. En estos pacientes en que la cisterna cuadrigeminal mostraba una morfología normal no había alteración del nivel de consciencia en ausencia de hidrocefalia, y se obtuvo una adecuada recuperación sin necesidad de evacuar el hematoma, lo que indicaba para los autores la ausencia de compresión suficiente del TDE como para producir un daño en el mismo. En aquellos casos en los que la cisterna apareció obliterada por completo, la puntuación GCS fue en todos menor o igual de 5, la hidrocefalia estuvo presente de forma constante y la evolución fue mala independientemente del tratamiento, quirúrgico o no. En los pacientes en 
que el hematoma provocaba una deformidad de la cisterna, sin llegar a ocluirla, se encontró un beneficio claro de la evacuación quirúrgica del hematoma y resolución de la hidrocefalia precoces ( $9 / 9$ pacientes experimentaron buena evolución), sufriendo aquellos casos en que no se realizó dicho tratamiento o se realizó tras 48 horas mala evolución (ninguno de los 2 pacientes no tratados, y sólo 2 de 5 pacientes operados más allá de 48 horas, experimentaron buena evolución). En los pacientes con deformidad Grado II sin hidrocefalia la puntuación GCS oscilaba entre 8 y 14, indicando suficiente compresión del tronco para causar disminución del nivel de consciencia. La variable que mejor se correlacionó con la evolución fue la deformidad de la cisterna cuadrigeminal, en tanto que el tamaño del hematoma se correlacionó con la evolución únicamente para sus valores más extremos. Experimentaron buena evolución un $88,4 \%$ de los pacientes con compresión grado I, un $68,8 \%$ de los pacientes con grado II y $0 \%$ de los pacientes con grado III.

- Kobayashi et $\mathrm{al}^{13}$, en un trabajo publicado en 1994 revisaron una serie retrospectiva de 52 pacientes con hematomas de cerebelo que fueron manejados de acuerdo a un protocolo por el que fueron intervenidos aquellos pacientes con lesiones de más de $3 \mathrm{~cm}$ de diámetro o más de 15 cc de volumen. Esta indicación no fue aplicada de forma estricta, lo que les permitió observar que en casos con hematomas de entre 3 y $4 \mathrm{~cm}$, en pacientes con buen nivel de consciencia se obtenía una evolución favorable con tratamiento conservador. Todos los pacientes con hematomas menores de $4 \mathrm{~cm}$ que fueron manejados conservadoramente experimentaron buena evolución. Por encima de este umbral únicamente un $16 \%$ de aquellos pacientes que se manejaron exclusivamente con tratamiento médico mostraron una recuperación adecuada, frente a un $60 \%$ de los que fueron operados. Además encontraron que en los pacientes con GCS $\leq 13$ el tratamiento conservador ofrecía una buena evolución en $12 \%$ de los casos frente a $46 \%$ del tratamiento quirúrgico. Globalmente un 54\% de los pacientes presentaron buena evolución, y un $27 \%$ fallecieron. Basados en estos hallazgos aplicaron de forma prospectiva en 49 pacientes un protocolo por el que operarían aquellos casos con hematomas mayores de $4 \mathrm{~cm}$ o $\mathrm{GCS} \leq 13$. Se desestimó el manejo agresivo en 9 pacientes en base a su edad, patología concomitante y situación clínica. De los 20 pacientes que inicialmente fueron seleccionados para tratamiento conservador todos experimentaron buena recuperación, aunque 2 precisaron ser intervenidos posteriormente (por crecimiento del hematoma e hidrocefalia progresiva respectivamente). De los 20 pacientes que cumplían criterios quirúrgicos 16 fueron operados, experimentando un $63 \%$ buena evolución. De los cuatro casos restantes en tres se optó por manejo alternativo dado que eran mayores de 70 años y presentaban adecuado nivel de consciencia, experimentando buena evolución tanto los dos manejados conservadoramente como el que fue tratado mediante aspiración del hematoma en fase subaguda. El paciente restante, con GCS 7, se trató únicamente mediante DVE, al presentar hidrocefalia y un hematoma de $3 \mathrm{~cm}$, y experimentó también buena recuperación. Los autores concluyeron recomendando el manejo conservador en pacientes con hematomas menores de $4 \mathrm{~cm}$ y GCS 14-15, en tanto que aquéllos con GCS $\leq 13$ o hematomas mayores de $4 \mathrm{~cm}$ serían los candidatos al tratamiento quirúrgico, salvo en caso de contraindicación del mismo por edad, antecedentes o mala situación clínica. Globalmente, y sin contar los pacientes en los que se desestimó manejo agresivo, un 85\% de los pacientes experimentó buena recuperación, con un $7,5 \%$ de mortalidad.

- Más tarde Kirollos et $a^{12}$, en ausencia de guías consistentes para el manejo de los hematomas de cerebelo decidieron emplear la morfología del IV ventrículo como principal criterio quirúrgico, retomando este dato descrito por Weisberg ${ }^{32}$. Además utilizaron el nivel de consciencia y la presencia de hidrocefalia como criterios adicionales de decisión terapéutica. Graduaron la compresión del IV ventrículo medido en el corte de mayor diámetro del hematoma en I: morfología y tamaño normales, localizado en línea media (en caso de hemorragia intraventricular se seguiría viendo LCR); II: IV ventrículo parcialmente comprimido o deformado, desviado, pero no ocluido; III: IV ventrículo colapsado por completo, con desviación anterior que distorsiona el tronco u obliteración de la cisterna prepontina (independientemente del diámetro ventricular). Todos los pacientes con compresión de grado III fueron tratados mediante evacuación del hematoma, al estimar que era indicación de una compresión grave del TDE. En el resto de casos el manejo se realizó en función del nivel de consciencia y la presencia o no de hidrocefalia. En aquellos casos con un IV ventrículo con grado I-II, hidrocefalia y deterioro del nivel de consciencia se realizó DVE. En los pacientes en grado II con mal nivel de consciencia, sin hidrocefalia o sin mejoría tras el tratamiento de la misma se realizó evacuación del hematoma. En el resto se realizó manejo conservador. Los autores analizaron la evolución de acuerdo a la escala de evolución de Glasgow a los tres meses. Se incluyeron 50 pacientes, 6 con grado I, 26 con grado II y 18 con grado III. Otros 6 pacientes fueron excluidos por mal grado clínico, grave enfermedad basal o por que el protocolo no se aplicó por otras razones. De los 6 pacientes con una compresión grado I, ninguno precisó la evacuación del hematoma, y todos experimentaron buena evolución. De los 9 pacientes con grado II que fueron manejados mediante observación, 5 experimentaron deterioro: uno de ellos falleció por TEP; tres de ellos fueron tratados mediante DVE experimentando mejoría y posteriormente buena evolución; uno no mostró mejoría tras 
DVE y requirió craniectomía de fosa posterior, con buena evolución posterior. De los 10 pacientes con compresión grado II inicialmente manejados con DVE, 3 precisaron craniectomía de fosa posterior ante la ausencia de mejoría. Entre los pacientes con compresión grado II, experimentaron buena evolución un $67 \%$ de los tratados mediante observación sólo, un $60 \%$ de los tratados mediante evacuación del hematoma y un $43 \%$ de los tratados mediante DVE. El $60 \%$ de los pacientes con hematomas $>3 \mathrm{~cm} \mathrm{y}$ compresión del IV ventrículo de grado I-II fueron tratados sin evacuación del hematoma, con manejo médico o DVE, mostrando un $67 \%$ de ellos buena evolución. Entre los 18 pacientes con compresión grado III un $67 \%$ mostraron deterioro clínico antes de poder ser tratados (un $43 \%$ de aquéllos con GCS $>9$ empeoró hasta el coma). Sólo un $17 \%$ de estos pacientes experimentó buena evolución. Se encontró una fuerte correlación entre el grado de deformación del IV ventrículo, el tamaño y la localización del hematoma y la presencia de hidrocefalia. Entre el conjunto de los que recibieron evacuación del hematoma la mortalidad fue del $54 \%$, frente a un $27 \%$ de los tratados mediante DVE. La mortalidad global fue del $40 \%$. El grado de deformidad del IV ventrículo y la situación clínica fueron los factores más estrechamente relacionados con la evolución.

A pesar de que se han publicado muchas otras series de pacientes tratados por hematomas de cerebelo, se centran fundamentalmente en datos clínicos y pronósticos, sin evaluar los criterios de indicación quirúrgica, por lo que no serán comentadas en este apartado.

\section{Discusión}

La correcta indicación quirúrgica en el hematoma de cerebelo puede conseguir, como ya se ha dicho, una elevada tasa de recuperación funcional (hasta un $44 \%{ }^{3,13}$ ) en pacientes que de otro modo, tendrían un mal pronóstico. Esta recuperación, que contrasta con la de los hematomas supratentoriales, se debe a la escasa elocuencia de la lesión que el abordaje quirúrgico ocasiona en el parénquima cerebeloso, y estaría condicionada a la ausencia de lesiones neurológicas irreversibles en el momento que se realiza el tratamiento. Sin embargo, el tratamiento quirúrgico no está exento de complicaciones ${ }^{12}$. La media de edad de estos pacientes es elevada y con frecuencia presentan comorbilidad significativa, con una mortalidad para los pacientes operados que oscila entre $34-64 \% \%^{4,7,12,20}$. Esto se debe en parte a que los pacientes intervenidos son aquéllos que parten de una peor situación clínica, pero el riesgo de la intervención debería ser evitado en pacientes que pueden evolucionar adecuadamente con tratamiento conservador o mediante el tratamiento aislado de la hidrocefalia, ya que en estos casos se alcanzan cifras de buena evolución del $91 \%{ }^{3}$. Por todo esto es necesario encontrar una pauta de tra- tamiento que permita la evacuación del hematoma en todos aquellos pacientes que lo precisen, evitando el riesgo de la cirugía en aquellos otros en los que no sea necesario este tratamiento. Esta pauta debe fundamentarse en unos criterios que hayan demostrado su utilidad en la discriminación entre estos grupos de pacientes.

Los criterios fundamentales para la decisión del tratamiento quirúrgico del hematoma de cerebelo, con aceptación universal son el nivel de consciencia y los signos tanto clínicos como radiológicos de compresión del tronco de encéfalo. El pronóstico de los pacientes con GCS $<14$ que se manejan de forma conservadora empeora respecto a aquéllos que son operados, por lo que hematomas que causan disminución del nivel de consciencia precisan tratamiento quirúrgico ${ }^{3,7,9,13,25,26,31}$. Sin embargo, la situación clínica no sirve como criterio único en la decisión del manejo de estos pacientes, ya que es insuficiente en la detección de aquellos casos que, como ya se ha comentado, pueden experimentar un deterioro a pesar de encontrarse alerta e íntegros neurológicamente en la valoración inicial. En este sentido son determinantes los datos que aporta la radiología referentes a las características del hematoma o su efecto sobre las estructuras vecinas. El tamaño del hematoma se correlaciona de forma directa con una peor situación clínica ${ }^{3,7}$. Tradicionalmente, y en gran parte fundamentado en el trabajo de Little et al ${ }^{14}$, se ha considerado que hematomas de más de $15 \mathrm{cc}$ de volumen o más de $3 \mathrm{~cm}$ de diámetro máximo debían ser evacuados quirúrgicamente, criterio que ha perdurado hasta ahora, casi 30 años después ${ }^{3-5,10,13,18,28,33}$. Sin embargo, se debe tener en cuenta que este fue uno de los primeros estudios con TC en esta patología, con las limitaciones inherentes a esta técnica para estudiar la fosa posterior. Además se incluyeron tan sólo 10 pacientes en aquel trabajo, y el papel que la hidrocefalia pudo tener en el grupo de pacientes con deterioro del nivel de consciencia fue obviado. Hay que recordar que en este grupo la presencia de ventriculomegalia fue constante, que uno de los pacientes con afectación del nivel de consciencia que fue operado presentaba un hematoma de menos de $3 \mathrm{~cm}$, y que en tres de los casos se observó hemorragia intraventricular, que podría contribuir a la obstrucción de LCR y al deterioro clínico. Así, muchos autores han observado después que pacientes con hematomas "grandes" y buena situación clínica mostraban un comportamiento clínico benigno sin ser precisa la intervención quirúrgica ${ }^{7,13,15,29}$. Kobayashi et al., revisaron este diámetro comprobando que el umbral que establecía la indicación quirúrgica podía elevarse a $4 \mathrm{~cm}$, obteniendo una evolución aceptable y evitando los riesgos de la intervención quirúrgica en pacientes que no la precisaban ${ }^{13}$. La ampliación del rango de pacientes que inicialmente podían manejarse de forma conservadora fue vista por algunos autores que comentaron dicho trabajo 
como una medida arriesgada, prefiriendo mantener un umbral quirúrgico más agresivo, aunque otros después han adoptado, al menos parcialmente, el umbral quirúrgico ampliado a 3,5-4 cm de diámetro máximo ${ }^{7,27,31}$. El emplear el diámetro del hematoma como criterio quirúrgico conlleva diversas limitaciones derivadas de su capacidad para estimar el grado de compresión del TDE. En primer lugar la valoración del mismo puede resultar confusa al definirse mal los límites de la lesión, y se puede ignorar el efecto de masa causado no por el coágulo en sí, sino por el conjunto del mismo y el edema circundante. Además, la compresión de las estructuras de fosa posterior no sólo depende del volumen ocupado por el hematoma y edema asociado, sino también de la relación entre estas estructuras y el espacio disponible en esta cavidad, con importante variabilidad interindividual. De este modo, hematomas del mismo volumen son mucho mejor tolerados en presencia de atrofia (como la atribuible a la edad o al efecto del consumo crónico de alcohol) y peor en el caso de fosas anatómicamente más estrechas. Por otro lado, el efecto de masa sobre el TDE varía también en función de la localización del hematoma. Hematomas del mismo diámetro ejercen mayor compresión cuando se localizan en línea media que cuando se localizan lateralmente dada la mayor proximidad del vermis al TDE. Todos estos problemas hacen que el diámetro del hematoma presente problemas de fiabilidad y tal vez sea sólo útil en sus valores extremos ${ }^{4,10,12,27,29}$.

Otros autores han investigado otros criterios alternativos que pudieran reflejar mejor el grado de compresión de las estructuras de fosa posterior. Weisberg ${ }^{32}$ consideró que la compresión de los espacios continentes de LCR de la fosa posterior y el desarrollo de hidrocefalia obstructiva, eran los mejores indicadores de la necesidad de la evacuación quirúrgica del hematoma ${ }^{32}$. En su serie encontró pacientes con hematomas de hasta $3,6 \mathrm{~cm}$ que evolucionaron adecuadamente sin cirugía, aunque otros casos con hematomas de $3 \mathrm{~cm}$ sí precisaron ser intervenidos, reflejando una escasa fiabilidad del diámetro del hematoma (no quedó bien definido sin embargo hasta qué punto algunos de los pacientes hubieran mejorado únicamente con tratamiento de la hidrocefalia asociada). De modo similar Taneda y cols ${ }^{29}$ estudiaron la compresión de la cisterna cuadrigeminal, encontrando una buena correlación de su afección con la evolución clínica. Teniendo en cuenta esta observación, propusieron un protocolo quirúrgico centrado en el grado de deformidad cuadrigeminal y en el manejo de la hidrocefalia. Este trabajo presentaba la importante limitación de no describir cuales fueron los criterios que rigieron el manejo de estos pacientes, además de que el estudio de la afección cuadrigeminal se realizó de forma retrospectiva. Aunque la correlación de la deformidad de esta cisterna con el pronóstico fue mejor que para el diámetro del hematoma, el método empleado para estimar el diámetro máximo fue diferente del empleado por otros autores. A pesar de que el valor pronóstico de la deformidad cuadrigeminal ha sido corroborado posteriormente ${ }^{30}$, otros autores han puesto de manifiesto las limitaciones en la interpretación de la morfología de la cisterna cuadrigeminal cuando puede verse ocupada por sangre y, sobre todo, cuando existe hidrocefalia $^{12}$. La presencia de dilatación ventricular conlleva un borramiento cisternal que, como los propios autores reconocieron $^{29}$, puede artefactar la utilidad de este parámetro como indicador de compresión del TDE. Además la afección de esta cisterna puede verse muy influenciada por la localización del hematoma; es posible que un hematoma vermiano origine una mayor deformidad de la cisterna que un hematoma hemisférico, aún con igual grado de compresión del TDE. Por último, dada la posición anatómica de la cisterna cuadrigeminal, su deformación podría ser únicamente representativa en casos de grave efecto de masa con herniación transtentorial. Dados los problemas tanto del volumen del hematoma como de la deformidad de la cisterna cuadrigeminal, Kirollos et al $^{12}$ emplearon el IV ventrículo como parámetro fundamental para la decisión terapéutica. Intuitivamente, esta estructura, quedando interpuesta entre el tejido hinchado por el hematoma, y la estructura cuya afección más preocupa, el TDE, podría reflejar de un modo directo el grado de compresión sobre éste. A pesar de que se observó una estrecha correlación entre el grado de deformidad ventricular y el pronóstico y la evolución clínica, el protocolo empleado resultó en una evolución poco satisfactoria en el grupo de pacientes con una deformidad intermedia de este ventrículo, deteriorándose un 55,6\% de los pacientes inicialmente manejados mediante observación y precisando evacuar el hematoma a la postre a un $30 \%$ de los pacientes en que en principio se decidió DVE. En éstos en que inicialmente no se realizó evacuación del hematoma se obtuvieron bajas tasas de buena recuperación ( $60 \%$ en los manejados mediante observación y 49\% en los manejados mediante DVE), cuando estos casos en principio debían partir de una buena situación clínica inicial, o al menos experimentar buena respuesta tras el DVE. Tanto los autores que propusieron este protocolo como otros ${ }^{12,34}$ recalcan lo delicado de los pacientes en el grado II que son manejados de forma conservadora o con drenaje ventricular externo. De hecho el grado de compresión del IV ventrículo puede ser un criterio insuficiente para decidir el manejo de estos pacientes en Grado II ${ }^{34}$.

La dilatación ventricular juega un papel indudable en el curso clínico del hematoma de cerebelo, está presente en $36-74 \%$ de los $\operatorname{casos}^{4,7,12,17,20,30}$, y presenta por sí misma relevancia pronóstica, habiendo centrado algunos autores el manejo quirúrgico de los hematomas de cerebelo en la combinación del nivel de consciencia y la hidrocefalia ${ }^{12,13,15,17}$. El grado en que la hidrocefalia es causada 


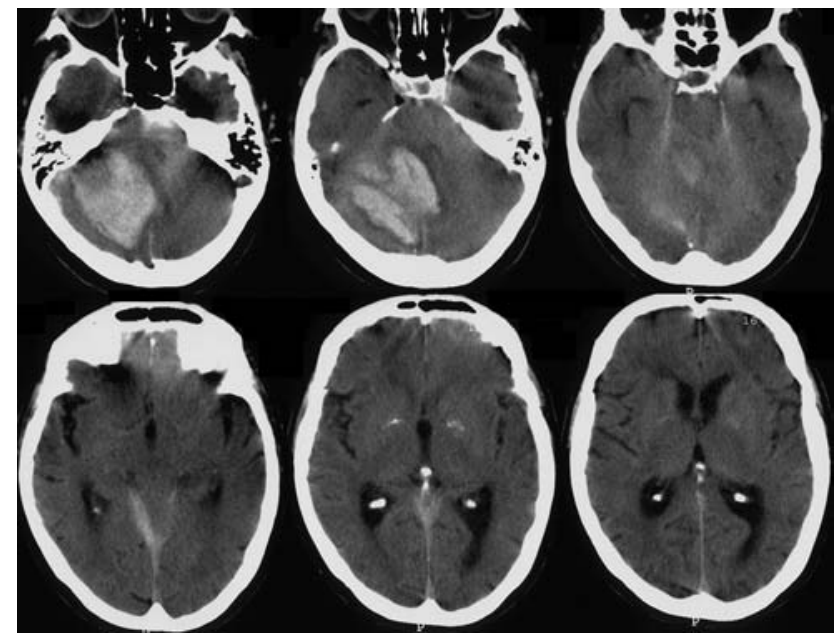

Figura 1. Mujer de 70 años, hipertensa, llevada al servicio de urgencias por cefalea, vómitos y deterioro del nivel de consciencia. En la valoración presentaba GCS 10 sin anomalías pupilares. La TAC mostró un hematoma de 5 $\mathrm{cm}$ de diámetro, con desplazamiento del IV ventrículo, oclusión de las cisternas prepontina y cuadrigeminal y leve dilatación ventricular. Se realizó una craniectomía de fosa posterior con evacuación del hematoma, tras lo que recuperó un adecuado nivel de consciencia. Fue dada de alta 11 dias tras la intervención, con ataxia truncal y en hemicuerpo derecho que mostraron una mejoría progresiva, deambulando con ayuda al mes de la intervención.

por compresión directa del TDE (y por tanto de las vías de circulación de LCR) es variable y así condiciona su implicación en el manejo propuesto por diversos autores. Algunos autores consideran la posibilidad de que la sangre intraventricular cause hidrocefalia obstructiva o que exista una compresión de las vías de LCR suficiente para obstruir su circulación pero insuficiente como para producir daño troncoencefálico ${ }^{12,13,29}$. La frecuencia con que se han observado estos pacientes en las diferentes series varía entre un $2-18 \%{ }^{12,13,17,30}$, y en ellos bastaría el drenado de LCR sin necesidad de evacuar el hematoma ${ }^{10,12,29}$. Otros autores han considerado que la dilatación ventricular se asocia constantemente a compresión del TDE y por lo tanto estiman que la hidrocefalia es indicación de la evacuación del hematoma ${ }^{4,9,12,14,17}$. Aunque algunos defienden la resolución de la hidrocefalia como tratamiento único inicial en todos los casos en que esté presente ${ }^{5,15,30}$, otros preconizan el drenado del hematoma y la descompresión de la fosa posterior siempre que se observe compresión de tronco, ya que aunque exista hidrocefalia concomitante el retraso en la evacuación del hematoma puede producir un deterioro irreversible , $, 3,7,9,12,13,17,26,34$. Aunque el desarrollo de una herniación infra-supratentorial ha sido considerada un evento infrecuente ${ }^{17,20,29}$, esta posibilidad no debe ser menospreciada $^{5,7,9,12,26,30}$.

Muchos autores subrayan el hecho de que el tratamiento de elección del hematoma de cerebelo está claro en los casos extremos ${ }^{3,12,13}$. Pacientes con afección del nivel de consciencia y hematomas grandes deben ser operados (Figura 1), y pacientes conscientes con hematomas pequeños no ${ }^{7}$. El manejo de los pacientes con buen nivel de consciencia y hematomas grandes, aquéllos con leve afección neurológica y hematomas de tamaño intermedio, o aquéllos con hematomas intermedios o grandes e hidrocefalia asociada es el que genera más problemas. La clave está en identificar el grado de compresión del TDE en cada caso y realizar un tratamiento lo más precoz posible a fin de evitar un daño neurológico no recuperable. Algunos autores abogan por el tratamiento quirúrgico incluso en pacientes conscientes si están presentes criterios radiológicos de riesgo dada la alta frecuencia de deterioro neurológico (67$75 \%$ ) y la disminución en la probabilidad de mejoría una vez que éste se ha producido $3,9,12,13,18,20,21,29$. Otros proponen una conducta expectante con observación estricta apoyándose en la escasa progresión clínica de pacientes en buena condición neurológica ${ }^{4,27}$. La frecuencia en que se presenta esta circunstancia parece variable, y en tanto que algunos autores la encuentran infrecuente ${ }^{27}$, otros la han observado en $22 \%$ de los pacientes incluidos en sus series ${ }^{3}$. En general se reconoce que ningún criterio por sí solo puede ofrecer un esquema que permita el manejo de todos los pacientes, y la mayoría de los protocolos de tratamiento se fundamenta en la combinación del nivel de consciencia con un criterio radiológico ${ }^{10,12,13,29}$. Aún así, existen casos límite, por las limitaciones que se han comentado tanto del tamaño del hematoma como de la afección de los espacios de LCR.

Con los datos disponibles, y a pesar de sus limitaciones, el volumen del hematoma y el grado de deformidad del IV ventrículo pueden ser los parámetros más fiables a la hora de decidir el mejor tratamiento de un paciente con un hematoma de cerebelo. El tamaño del hematoma es el parámetro que ha sido empleado con mayor frecuencia como criterio radiológico para la indicación de cirugía, en tanto que dentro de los indicadores de "compresión de la fosa posterior", la deformidad del IV ventrículo ha sido el mejor evaluado prospectivamente como un índice independiente. En presencia de un hematoma mayor de $4 \mathrm{~cm}$ de diámetro o de una compresión marcada del IV ventrículo (grado III) el tratamiento debería ser quirúrgico, incluso en pacientes alerta, ya que la probabilidad de deterioro del paciente a pesar de presentar un adecuado nivel de consciencia inicial es muy alta, puede darse de forma abrupta y el retraso en el tratamiento una vez que se produce el empeoramiento puede resultar fata ${ }^{12,20}$ (Figura 2). Dicha evacuación puede ser complementada con un drenaje ventricular externo en el mismo acto quirúrgico cuando exista hidrocefalia asociada, evitando una evacuación excesiva de LCR que 
Pérez-Núñez y col

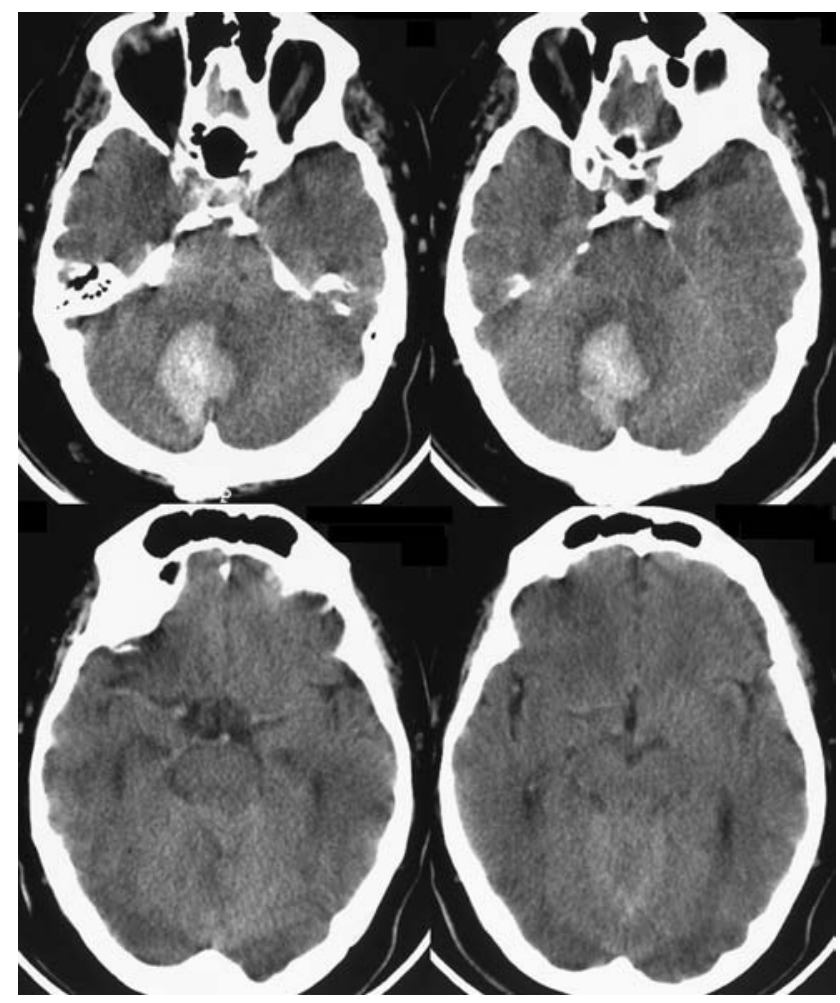

Figura 2. Paciente de 42 años, obeso mórbido e hipertenso. Valorado tras 6 días de mareo, vómitos y torpeza en hemicuerpo derecho con marcha imposible. Presentaba una puntuación en la Escala de Coma de Glasgow de 15 y signos cerebelosos y Babinski derechos. Se diagnosticó un hematoma cerebeloso de $4,5 \mathrm{~cm}$, con deformidad grado II del IV ventrículo y cisterna cuadrigeminal, y borramiento de la cisterna prepontina. Fue tratado con craniectomía y evacuación del hematoma y dado de alta hospitalaria a los 21 días con recuperación completa salvo por leve torpeza en la mano derecha.

favorezca el desarrollo de una herniación cerebelosa infrasupratentorial $^{12,13}$. Con hematomas menores de $3 \mathrm{~cm}$ de diámetro máximo y que no afectan al tamaño ni morfología del IV ventrículo, el manejo inicial debe ser conservador, o se debe realizar DVE si existe hidrocefalia que cause deterioro del nivel de consciencia. Existe un territorio de incertidumbre, con hematomas entre 3 y $4 \mathrm{~cm}$ ó compresión del tronco en grado II (presente, pero no grave). En estos casos intermedios, estando ausente cualquiera de los criterios definitivos de compresión del TDE, parece que estaría justificada una actitud expectante cuando el nivel de consciencia esté conservado, y también un tratamiento exclusivo inicial de la hidrocefalia si éste fuera preciso (Figuras 3 y 4). En este grupo de pacientes existe más controversia entre los que propugnan una actitud más agresiva y aquéllos que prefieren mantener un manejo más conservador. La combinación del diámetro del hematoma y de la morfología del IV ventrículo como criterios de deci-
Neurocirugía 2008; 19: 101-112

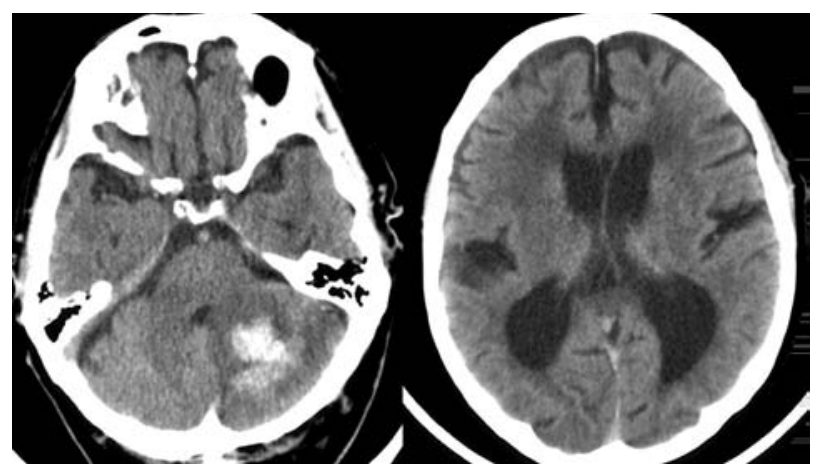

Figura 3. Paciente de 83 años hipertenso, nefrópata, con fibrilación auricular e insuficiencia cardiaca. Consultó por clínica de 4 días de cefalea y vómitos que después asociaron imposibilidad para caminar, torpeza en la manipulación e incontinencia de orina. Presentaba una puntuación de 15 según la Escala de Coma de Glasgow sin focalidad neurológica, y la TAC diagnosticó un hematoma de cerebelo de 3,7 cm de diámetro. Se realizó tratamiento conservador con mejoría progresiva, hasta que el paciente pudo deambular con apoyo y ser dado de alta el 14 día de ingreso.

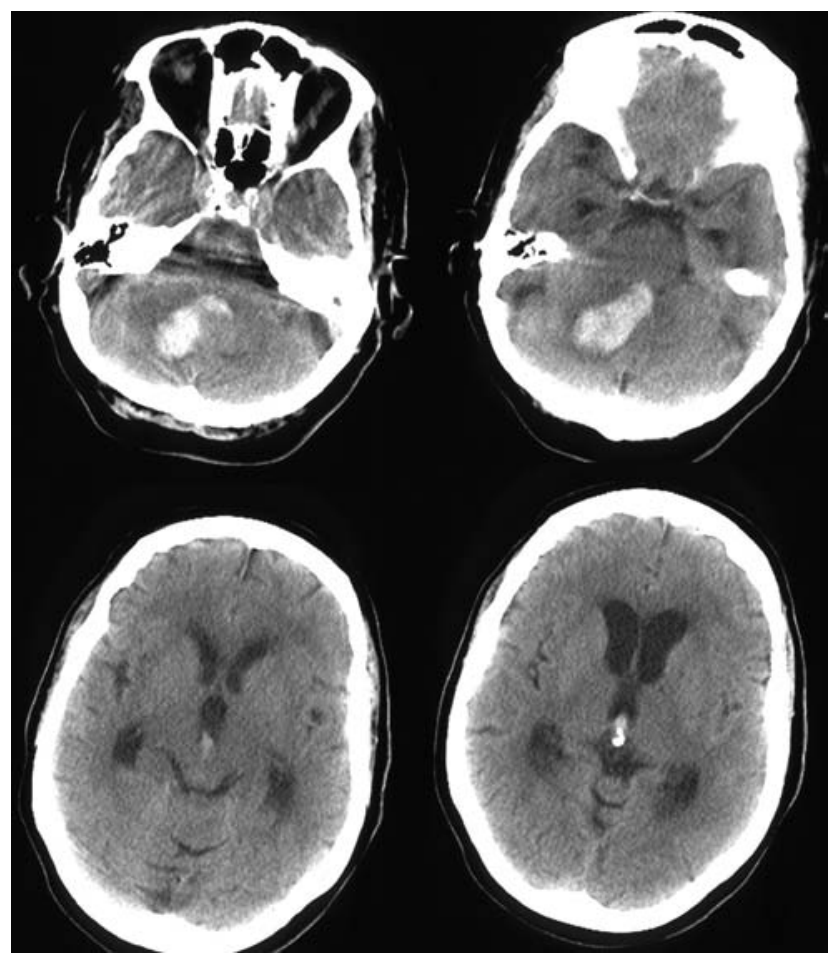

Figura 4. Mujer de 79 años hipertensa, que fue llevada a urgencias por vómitos y progresiva disminución del nivel de consciencia. A su ingreso abría los ojos y presentaba respuesta de localización con extremidades al estímulo doloroso, sin respuesta verbal. La TAC mostró un hematoma cerebeloso de 3,6 cm de diámetro, sangrado intraventricular e hidrocefalia asociada. Se colocó un drenaje ventricular externo durante 6 dias, con buena evolución posterior y alta hospitalaria a los 21 dias caminando con ayuda. 


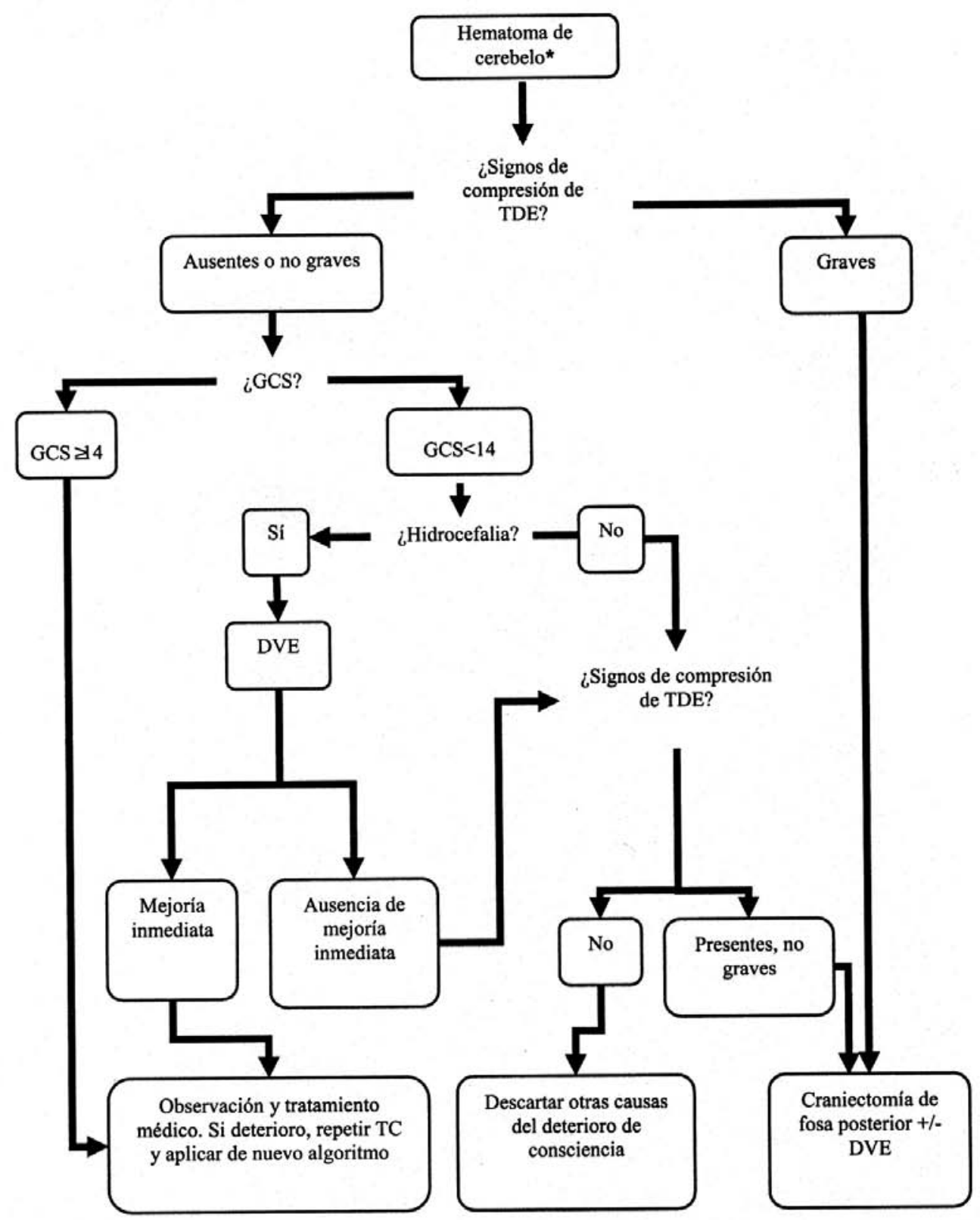

Figura 5. Algoritmo de manejo quirúrgico del hematoma de cerebelo.

DVE: Drenaje ventricular externo. GCS: Escala de Coma de Glasgow. TDE: Tronco de encéfalo.

Signos de compresión del tronco de encéfalo:

1. Ausentes: diámetro del hematoma $<3 \mathrm{~cm}$ y IV ventrículo normal.

2. Presentes, no graves: diámetro del hematoma $\geq 3 \mathrm{~cm}$ y $<4 \mathrm{~cm}$ y/o IV ventrículo parcialmente deformado, comprimido o desviado de la línea media.

3. Graves: diámetro del hematoma $\geq 4 \mathrm{~cm}$, IV ventrículo colapsado o compresión anterior del tronco con deformación del mismo u obliteración de la cisterna prepontina.

*No serán incluidos en este protocolo pacientes que por su grave situación basal, avanzado deterioro neurológico, afección del tronco de encéfalo o avanzada edad presenten un pronóstico fatal. 
sión puede aumentar la seguridad del manejo, al compensar las limitaciones individuales de cada índice como indicador de la compresión del TDE. Salvatti et a ${ }^{27}$ ya emplearon un método similar, en el que el parámetro principal de decisión terapéutica era el diámetro del hematoma pero se variaba el umbral quirúrgico en función de la localización del hematoma y la presencia o no de signos de "compresión de la fosa posterior". Así, en tanto que el límite se establecía de forma genérica en $4 \mathrm{~cm}$, para los hematomas vermianos era de 3,5 cm y para los que originaban signos de "compresión de la fosa posterior" era de $3 \mathrm{~cm}$. Con esto obtuvieron una supervivencia del 59\% y una proporción de buena evolución del 45,4\% entre los pacientes supervivientes. En cualquier caso, ante la duda, en presencia de empeoramiento tras manejo conservador o ante la ausencia de mejoría tras el tratamiento de la hidrocefalia, se debería realizar una descompresión con evacuación del hematoma lo antes posible. Existe acuerdo en que el retraso en el tratamiento (bien de la hidrocefalia o la evacuación del hematoma) de los pacientes que lo precisan, disminuye las posibilidades de una recuperación adecuada ${ }^{3,29}$. Los supuestos comentados anteriormente y un posible algoritmo de decisión terapéutica se reflejan en la figura 5 .

A pesar de los buenos resultados del tratamiento quirúrgico en esta patología aquellos pacientes con tetraplejia fláccida y ausencia de reflejos de TDE, o aquéllos con un deterioro grave de su calidad de vida previa $(\mathrm{KPS}<70)$ o con enfermedades graves que contraindique el tratamiento quirúrgico probablemente no van a obtener beneficio con el mismo ${ }^{4,7,13,27}$. Sin embargo, algunos pacientes en coma pueden alcanzar elevadas tasas de recuperación ${ }^{3,9,21}$, si bien la mortalidad en ellos oscila entre $35-100 \%$ (88.9$100 \%$ en presencia de coma profundo) $)^{17,20,35}$. La cirugía por tanto, podría no ofrecer beneficio en caso de deterioro neurológico avanzado, sobre todo cuando se observan graves signos de compresión del TDE ${ }^{29,35}$. Aún así, algunos autores propugnan extender la indicación de este tratamiento al extremo clínico de los pacientes con mayor deterioro, incluso en midriasis arreactiva bilateral. Los datos en que se apoyan algunos de ellos no parecen tan optimistas como la tesis que defienden. Yanaka et $\mathrm{al}^{35}$ intervinieron a 16 pacientes con GCS 3 y anomalías pupilares. Tres de ellos se recuperaron hasta un grado de discapacidad moderada, pudiendo dos de ellos volver a trabajar; tres pacientes evolucionaron a discapacidad grave, cuatro quedaron en estado vegetativo persistente y 6 murieron. Un $15 \%$ de los pacientes experimentó buena evolución. El único factor que fue significativamente diferente entre aquéllos con buena o mala evolución fue que en los primeros la intervención se llevó a cabo antes de 2 horas tras el ictus. La condición de un tratamiento precoz tras el deterioro es condición indispensable para otros autores que propugnan la intervención en los pacientes en coma profundo ${ }^{9,21}$. Una postura empleada por algunos autores en casos de pacientes de mal pronóstico, por una mala situación previa o por presentarse en coma profundo, era el evitar la evacuación del hematoma y realizar un DVE, intervención de menor riesgo ${ }^{5,17}$. La mejoría tras este procedimiento podría derivar en la realización de una evacuación del hematoma, al apuntar a un mejor pronóstico por la ausencia de lesiones establecidas en el TDE ${ }^{17}$. Diversos autores han observado que pacientes en coma $(\mathrm{GCS}<5-9)$ con hematomas voluminosos o signos graves de compresión del TDE en TAC experimentaron de forma constante una evolución adversa, expresando sus dudas en cuanto a la indicación de evacuar el hematoma en estos $\operatorname{casos}^{12,16,29,31}$. Se reconoce sin embargo, la necesidad de tratar a aquellos pacientes con hidrocefalia y sin signos de grave compresión del TDE independientemente de la gravedad del coma, estimando que la evacuación de la hidrocefalia puede provocar una dramática mejoría ${ }^{29}$. Si bien no creemos indicado abandonar el tratamiento quirúrgico sistemáticamente en pacientes con GCS $\leq 8$ en presencia de datos de compresión de TDE, tampoco estimamos que haya datos a favor de tratar a pacientes con GCS 3 y ausencia de reflejos de TDE.

Aunque no hemos encontrado ningún ensayo clínico que compare la utilidad de la cirugía con la del tratamiento médico, varios estudios observacionales indican que dicho ensayo no estaría justificado éticamente, al menos en el conjunto de estos pacientes, ya que el beneficio de la descompresión quirúrgica en algunos de ellos está fuera de duda $^{2,4,6,24,25}$. La escasa incidencia de este proceso hace que las series publicadas sean pequeñas, y las recomendaciones para el tratamiento en general se apoyan en los resultados de éstas o en la experiencia individual de los cirujanos que las reportan ${ }^{3,33}$. La disparidad de criterios quirúrgicos y de los pacientes incluidos desemboca en diversos sesgos que en ocasiones hacen difícil comparar los estudios entre sí. No existen aún guías de manejo universalmente aceptadas, por lo que la elaboración de un protocolo terapéutico que fuera periódicamente evaluado tras ser aplicado de forma prospectiva, permitiendo así su perfeccionamiento, ayudaría a mejorar el manejo de estos pacientes en los que la intervención quirúrgica puede marcar la diferencia entre una evolución fatal o la incapacidad permanente, y una vida prácticamente normal. Cabe preguntarse sobre la posibilidad de desarrollar un ensayo clínico en el grupo de pacientes cuya situación se encuentra en un territorio de incertidumbre, en los que como se ha comentado las indicaciones en cada centro son variables.

\section{Bibliografía}

1. Auer, L.M., Deinsberger, W., Niederkorn, K., et al.: Endoscopic surgery versus medical treatment for spontaneous intracerebral hematoma: a randomized study. J Neurosurg 
1989; 70: 530-535.

2. Broderick, J.P., Adams, H.P., Jr., Barsan, W., et al.: Guidelines for the management of spontaneous intracerebral hemorrhage: A statement for healthcare professionals from a special writing group of the Stroke Council, American Heart Association. Stroke 1999; 30: 905-915.

3. Cohen, Z.R., Ram, Z., Knoller, N., Peles, E., Hadani, M.: Management and outcome of non-traumatic cerebellar haemorrhage. Cerebrovasc Dis 2002; 14: 207-213.

4. Dolderer, S., Kallenberg, K., Aschoff, A., Schwab, S., Schwarz, S.: Long-term outcome after spontaneous cerebellar haemorrhage. Eur Neurol 2004; 52: 112-119.

5. Donauer, E., Loew, F., Faubert, C., Alesch, F., Schaan, M.: Prognostic factors in the treatment of cerebellar haemorrhage. Acta Neurochir (Wien ) 1994; 131: 59-66.

6. Fewel, M.E., Thompson, B.G., Jr., Hoff, J.T.: Spontaneous intracerebral hemorrhage: a review. Neurosurg Focus [Revista electrónica] 2003; 15(4): Artículo 1 [16 p]. Disponible en: http://www.aans.org/education/journal/neurosurgical/ oct03/15-4-1.pdf.

7. González-García, J., Gelabert-Gonzalez, M., GarcíaAllut, A., Fernández-Villa, J.M., López-García, E., GarcíaPravos, A.: Cerebellar hematomas: a surgically treatable stroke. Rev Neurol 2000; 31: 1119-1126.

8. Heiskanen, O.: Treatment of spontaneous intracerebral and intracerebellar hemorrhages. Stroke 1993; 24: I94I95.

9. Heros, R.C.: Cerebellar hemorrhage and infarction. Stroke 1982; 13: 106-109.

10. Jensen, M.B., St Louis, E.K.: Management of acute cerebellar stroke. Arch Neurol 2005; 62: 537-544.

11. Kanaya, H., Kuroda, K.: Development in neurosurgical approaches to hypertensive intracerebral hemorrhage in Japan. En Kaufmann, H.H. (ed). Intracerebral Hematomas. New York; Raven Press Ltd, 1992; pp.197210.

12. Kirollos, R.W., Tyagi, A.K., Ross, S.A., van Hille, P.T., Marks, P.V.: Management of spontaneous cerebellar hematomas: a prospective treatment protocol. Neurosurgery 2001; 49: 1378-1386.

13. Kobayashi, S., Sato, A., Kageyama, Y., Nakamura, H., Watanabe, Y., Yamaura, A.: Treatment of hypertensive cerebellar hemorrhage--surgical or conservative management? Neurosurgery 1994; 34: 246-250.

14. Little, J.R., Tubman, D.E., Ethier, R.: Cerebellar hemorrhage in adults. Diagnosis by computerized tomography. J Neurosurg 1978; 48: 575-579.

15. López-Ferro, M.O., Gómez López, P.A., Muniz, d., I, Mestre, M.C., Queimadelos, Boixados, S., Jr.: Conservative treatment of spontaneous hematomas of the cerebellum. Rev Clin Esp 1985; 176: 169-172.

16. Luparello, V., Canavero, S.: Treatment of hypertensive cerebellar hemorrhage: surgical or conservative management?
Neurosurgery $1995 ; 37: 552-553$.

17. Mathew, P., Teasdale, G., Bannan, A., OluochOlunya, D.: Neurosurgical management of cerebellar haematoma and infarct. J Neurol Neurosurg Psychiatry 1995; 59: 287-292.

18. Mayer, S.A., Rincon, F.: Treatment of intracerebral haemorrhage. Lancet Neurol 2005; 4: 662-672.

19. McKissock, W., Richardson, A., Walsh, L.: Spontaneous cerebellar hemorrhage: a study of 34 consecutive cases treated surgically. Brain 1960; 83: 1-9.

20. Mezzadri, J.J., Otero, J.M., Ottino, C.A.: Management of 50 spontaneous cerebellar haemorrhages. Importance of obstructive hydrocephalus. Acta Neurochir (Wien ) 1993; 122: 39-44.

21. Ojemann, R.G., Heros, R.C.: Spontaneous brain hemorrhage. Stroke 1983; 14: 468-475.

22. Pérez, A., Díez, R.: Tratamiento quirúrgico de la hemorragia intracerebral espontánea. En Net, Á., Marruecos-Sant, L. (ed). El Paciente Neurocrítico. Barcelona; Ars Medica, 2006; pp. 273-284.

23. Pollak, L., Rabey, J.M., Gur, R., Schiffer, J.: Indication to surgical management of cerebellar hemorrhage. Clin Neurol Neurosurg 1998; 100: 99-103.

24. Prasad, K., Shrivastava, A.: Surgery for primary supratentorial intracerebral haemorrhage (Cochrane Review). En The Cochrane Lybrary, Volumen 1. Chichester, UK; John Wiley \& Sons, Ltd, 2004.

25. Qureshi, A.I., Tuhrim, S., Broderick, J.P., Batjer, H.H., Hondo, H., Hanley, D.F.: Spontaneous intracerebral hemorrhage. N Engl J Med 2001; 344: 1450-1460.

26. Salazar, J., Vaquero, J., Martínez, P., Santos, H., Martínez, R., Bravo, G.: Clinical and CT scan assessment of benign versus fatal spontaneous cerebellar haematomas. Acta Neurochir (Wien ) 1986; 79: 80-86.

27. Salvati, M., Cervoni, L., Raco, A., Delfini, R.: Spontaneous cerebellar hemorrhage: clinical remarks on 50 cases. Surg Neurol 2001; 55: 156-161.

28. Siddique, M.S., Mendelow, A.D.: Surgical treatment of intracerebral haemorrhage. Br Med Bull 2000; 56: 444456.

29. Taneda, M., Hayakawa, T., Mogami, H.: Primary cerebellar hemorrhage. Quadrigeminal cistern obliteration on CT scans as a predictor of outcome. J Neurosurg 1987; 67: 545552.

30. Van Loon, J., Van Calenbergh, F., Goffin, J., Plets, C.: Controversies in the management of spontaneous cerebellar haemorrhage. A consecutive series of 49 cases and review of the literature. Acta Neurochir (Wien ) 1993; 122: 187-193.

31. Vanaclocha, V., Saiz-Sapena N., Kay de Villiers, J.: Hematomas cerebelosos. Neurocirugía 1998; 9: 26-34.

32. Weisberg, L.A.: Acute cerebellar hemorrhage and CT evidence of tight posterior fossa. Neurology 1986; 36 : 
858-860.

33. Wijdicks, E.F., St Louis, E.K., Atkinson, J.D., Li, H.: Clinician's biases toward surgery in cerebellar hematomas: an analysis of decision-making in 94 patients. Cerebrovasc Dis 2000; 10: 93-96.

34. Yanaka, K., Matsumaru, Y., Nose, T.: Management of spontaneous cerebellar hematomas: a prospective treatment protocol. Neurosurgery 2002; 51: 524-525.

35. Yanaka, K., Meguro, K., Fujita, K., Narushima, K., Nose, T.: Immediate surgery reduces mortality in deeply comatose patients with spontaneous cerebellar hemorrhage.
Neurol Med Chir (Tokyo) 2000; 40: 295-299.

Pérez-Núñez, A.; Alday, R.; Rivas, J.J.; Lagares, A.; Gómez, P.A.; Alén, J.F.; Arrese, I.; Lobato, R.D.: Tratamiento quirúrgico de la hemorragia intracerebral espontánea. Parte II: Hemorragia infratentorial. Neurocirugía 2008; 19: 101-112.

Correspondencia postal: Ángel Pérez Núñez. Servicio de Neurocirugía. Hospital Doce de Octubre. Avda de Córdoba s/n. 28041 Madrid 